Bull. Mater. Sci., Vol. 16, No. 3, June 1993, pp. 187-191. O Printed in India.

\title{
Electrical and optical properties of molybdenum trioxide thin films
}

\author{
S KRISHNAKUMAR and C S MENON
}

School of Pure and Applied Physics, Mahatma Gandhi University, Ettumanoor, Kottayam 686631, India

MS received 27 April 1992; revised 12 March 1993

\begin{abstract}
Infrared spectra of vacuum-deposited molybdenum trioxide thin films have been studied. The variation of electrical conductivity with temperature for different thicknesses of films has been investigated. Electrical conductivity of the films as a function of time of UV irradiation was found to increase initially, then decreased rapidly and reached a steady value. It increased and reached a steady value with time when irradiation was cut-off.
\end{abstract}

Keywords. Molybdenum trioxide; thin films; IR spectra; activation energy; UV irradiation.

\section{Introduction}

Molybdenum trioxide $\left(\mathrm{MoO}_{3}\right)$ is a transition metal oxide having a number of electrical and optical properties. Lampert (1984) reviewed numerous inorganic and organic electrochromic materials in the context of developing a film-based optical shutter for energy-efficient windows. Thin films of $\mathrm{MoO}_{3}$ can be used in electronic information displays and colour memory devices. The last decade saw extensive electrical and optical investigation of electrochromic materials (Yoshimura et al 1983; Donnadieu and Davazoglou 1986; Davazoglou and Donnadieu 1987; Davazoglou et al 1987).

Coloured centres in thin films of $\mathrm{MoO}_{3}$ can be formed by UV light irradiation. Deb and Chopoorian (1966) reported that the colour centres formed as a result of free electrons being trapped at oxygen ion vacancies. The mechanism behind this colouration is not yet well understood. In this paper we report IR spectra, activation energy of $\mathrm{MoO}_{3}$ films and variation of its electrical conductivity during and after UV irradiation.

\section{Experimental}

The films of $\mathrm{MoO}_{3}$ were prepared by physical vapour deposition using a thermal evaporation plant (Hind Hivac, model:12 A4) which provided a base pressure of $4 \times 10^{-4} \mathrm{~Pa} . \mathrm{MoO}_{3}(99.9 \%$ pure) (Goodfellow, UK) was evaporated from a tantalum boat on to the mylar and glass substrates kept above the source. A constant rate of evaporation was maintained which was monitored by a quartz crystal thickness monitor having an accuracy of $1 \mathrm{~nm}$. The value thus obtained by thickness monitor was counterchecked by using the Fizeau fringe technique (Chopra 1969). $\mathrm{MoO}_{3}$ films on mylar substrate was used for IR studies (IR spectrophotometer, Shimadzu IR 470). Keithley 617 programmable electrometer was used for studying d.c. electrical conductivity by varying temperature of the films on glass substrate having a fixed bias voltage of $0.8 \mathrm{~V}$. The d.c. electrical conductivity of the film on a glass substrate was also measured using an electrometer by applying a fixed bias voltage of $0.8 \mathrm{~V}$ to the film at room temperature during and after UV irradiation. 


\section{Results and Discussion}

The transmission spectra of $\mathrm{MoO}_{3}$ film formed on mylar (polyethylene terephthalate) substrate were recorded in IR (4000 to $400 \mathrm{~cm}^{-1}$ ) region by an infrared spectrophotometer with a similar uncoated mylar as a reference. Most of the molecular vibrational frequencies were present in this region. Figure 1 gives the IR spectra of $\mathrm{MoO}_{3}$ film of thickness $134 \mathrm{~nm}$ on a mylar sheet. The notable features of the $\mathrm{MoO}_{3}$ spectra are the weak transmission bands observed at 580 and $780 \mathrm{~cm}^{-1}$ and the strong transmission bands at 468,890 and $1000 \mathrm{~cm}^{-1}$. These values are in agreement with the reported peaks at 468,620,810,890 and $1020 \mathrm{~cm}^{-1}$ (Eda 1989; Anwar et al 1990).

Figure 2 shows the temperature dependence of d.c. electrical conductivity $(\sigma)$ of the films when a fixed bias voltage of $0.8 \mathrm{~V}$ is applied for three different thicknesses 165, 187 and $208 \mathrm{~nm}$ respectively. Assuming an Arrhenius-type relation,

$$
\sigma=\sigma_{0} \exp (-\Delta E / k T)
$$

where $\sigma_{0}$ is the pre-exponential factor and $k$, the Boltzmann constant and $\Delta E$, the thermal activation energy. The plot $\ln \sigma$ vs $\left(10^{3} / T\right)$ exhibits two straight line regions of different activation energies of conductivity for the same $\mathrm{MoO}_{3}$ sample. The value of activation energy $\Delta E_{1}$ in the lower temperature region as well as activation energy $\Delta E_{2}$ in the higher temperature region show slight change with thickness of the film.

The variation of d.c. electrical conductivity as a function of irradiation time of $\mathrm{MoO}_{3}$ thin film of thickness $208 \mathrm{~nm}$ is shown in figure 3 . Here the d.c. electrical conductivity was measured while increasing the irradiation time. It was seen that the conductivity increased initially, and then decreased much faster. After $30 \mathrm{~min}$, it reached almost a steady value. Due to colouration, the films changed from greenishblue to dark blue in colour. Figure 4 gives the variation of conductivity as a function

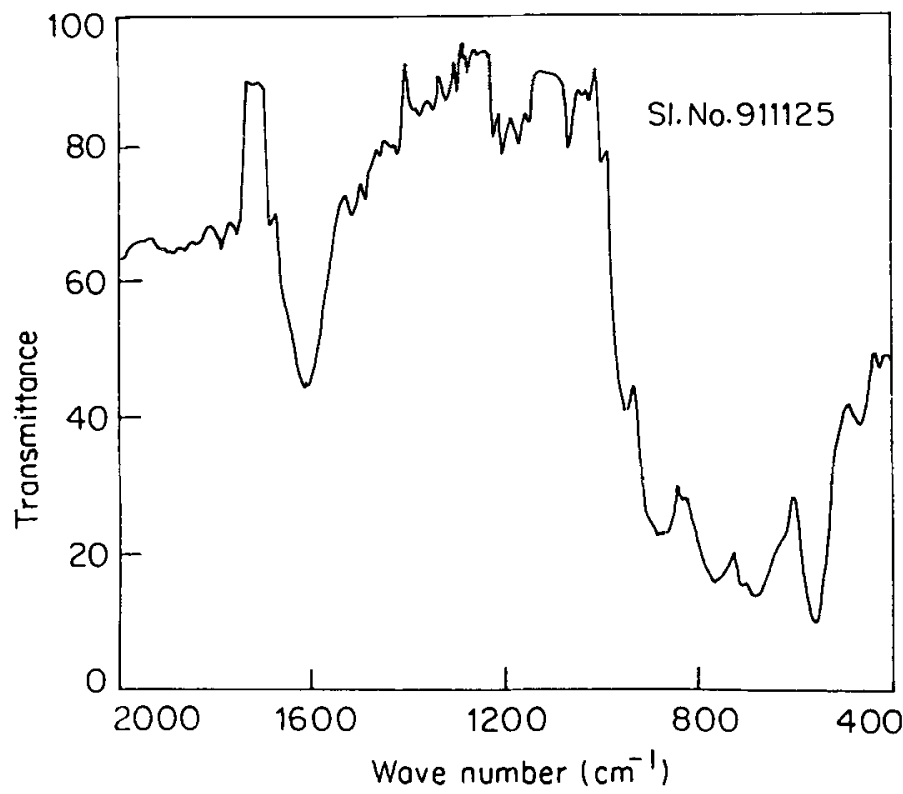

Figure 1. Infrared spectra of $\mathrm{MoO}_{3}$ thin films of thickness $134 \mathrm{~nm}$. 


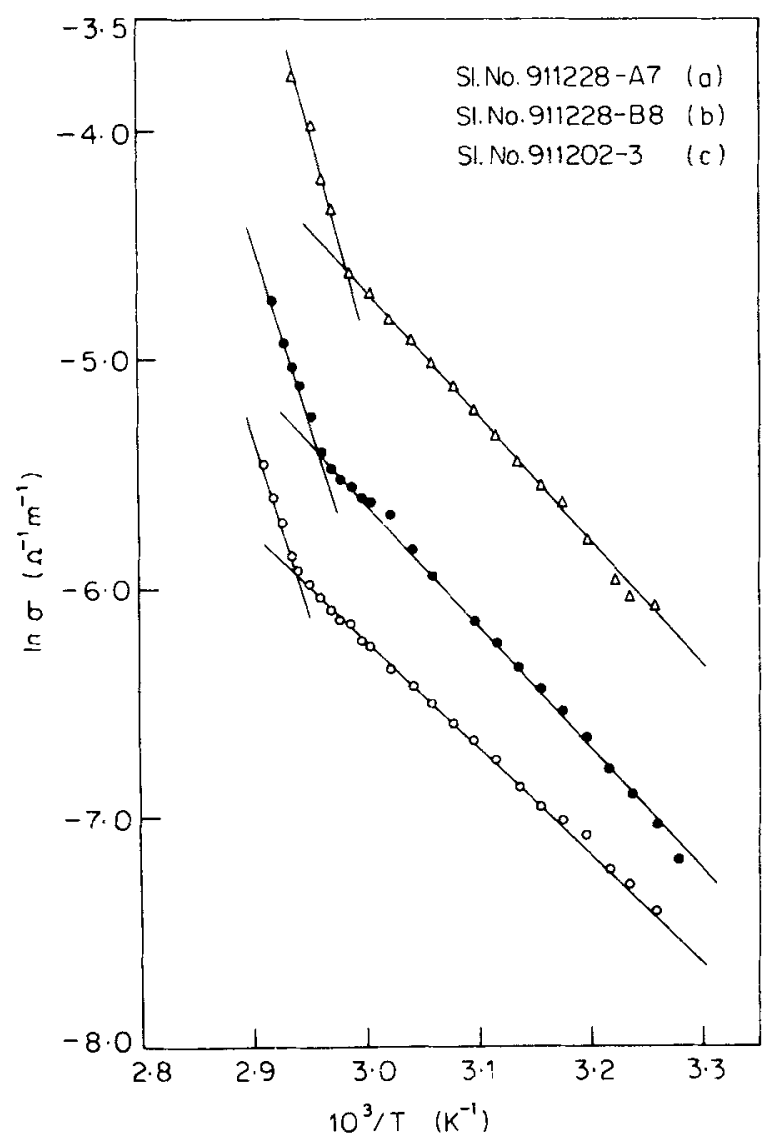

Figure 2. Temperature variation of d.c. electrical conductivity of $\mathrm{MoO}_{3}$ films of thicknesses (a) $165 \mathrm{~nm}$. (b) $187 \mathrm{~nm}$ and (c) $208 \mathrm{~nm}$.

Table 1. Activation energies $\Delta E_{1}$ and $\Delta E_{2}$ obtained for different thicknesses and $\mathrm{MoO}_{3}$ films.

\begin{tabular}{|c|c|c|c|}
\hline $\begin{array}{l}\text { Sample } \\
\text { No. }\end{array}$ & $\begin{array}{c}\text { Thickness } \\
\text { (nm) }\end{array}$ & $\begin{array}{c}\Delta E_{1} \\
(305-338 \mathrm{~K}) \\
(\mathrm{eV})\end{array}$ & $\begin{array}{c}\Delta E_{2} \\
(338-348 \mathrm{~K}) \\
(\mathrm{eV})\end{array}$ \\
\hline 911228-A7 & 165 & $0-46$ & $1 \cdot 35$ \\
\hline 911228-B8 & 187 & 0.43 & $1 \cdot 32$ \\
\hline $911202-3$ & 208 & 0.41 & $1 \cdot 29$ \\
\hline
\end{tabular}

of time after terminating the irradiation. It was found that conductivity increased much faster at the beginning and reached a steady value.

The conductivity of transition metal oxides was due to the hopping of small polarons (Austin and Mott 1969). When we irradiated $\mathrm{MoO}_{3}$ thin film with UV light, initially its conductivity increased. As a result of irradiation of the film, the phonons took the energy which resulted in the electron-phonon interaction, so that the effective mass of the polaron increased. Since the effective mass increased, the resistivity also 


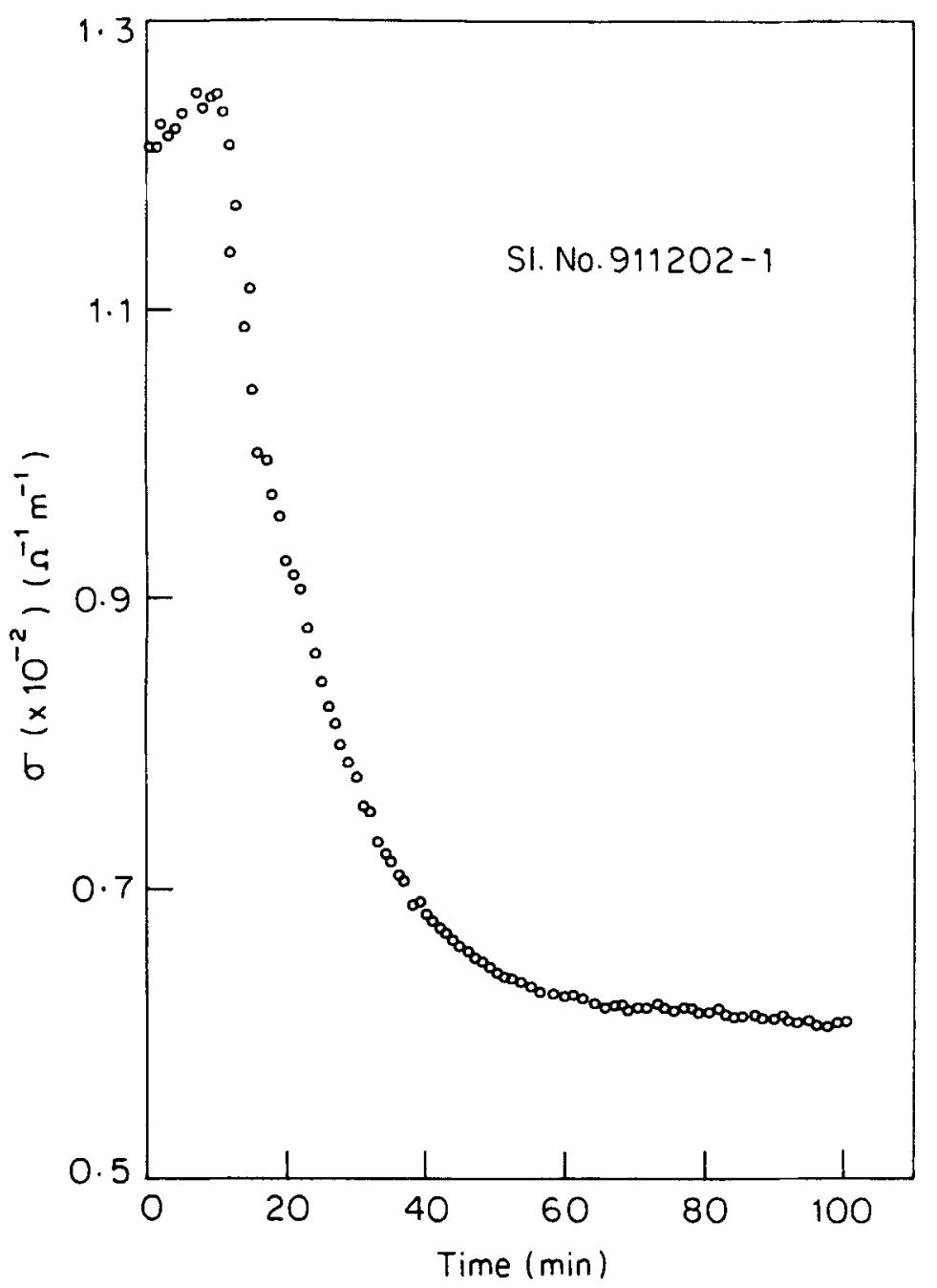

Figure 3. Variation of d.c. electrical conductivity as a function of time while UV irradiating $\mathrm{MoO}_{3}$ films of thickness $208 \mathrm{~nm}$.

increased, which in turn decreased its conductivity. After some time the electronphonon interaction reached an equilibrium. As a consequence, a steady value of conductivity was reached. From figure 3 it is seen that cutting-off the irradiation terminated electron-phonon interaction. The conductivity rapidly increased and reached a steady value. This suggests that the electrical properties of $\mathrm{MoO}_{3}$ are sensitive to high energy electromagnetic radiation and time of exposure.

\section{Conclusion}

From IR spectrum it is clear that the film formed was $\mathrm{MoO}_{3}$. The film was capable of colouring by UV irradiation. Activation energy of conductivity for $\mathrm{MoO}_{3}$ thin film varied with thickness of the film. The d.c. electrical conductivity of $\mathrm{MoO}_{3}$ film 


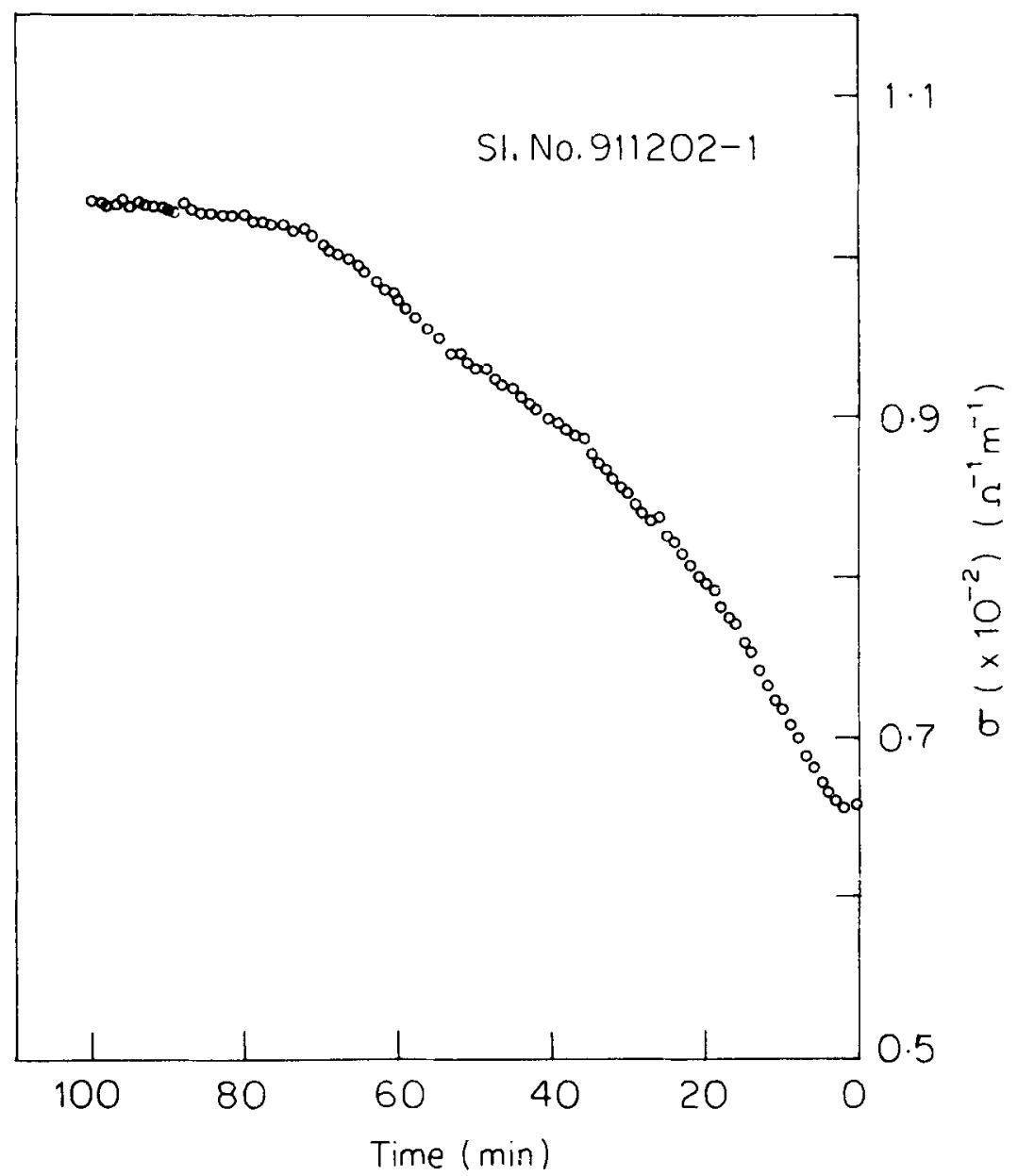

Figure 4. Variation of d.c. electrical conductivity as a function of time after terminating UV irradiation of $\mathrm{MoO}_{3}$ film of thickness $208 \mathrm{~nm}$.

initially increased and then decreased and finally reached a steady value during UV irradiation. It increased rapidly and reached a steady value when the irradiation was stopped.

\section{References}

Anwar M, Hogarth C A and Theocharis C R 1990 J. Mater. Sci. 241108 Austin I G and Mott N F 1969 Adv. Phys. 1841

Chopra K L 1969 Thin film phenomena (New York: McGraw Hill) p 103

Davazoglou D, Donnadieu A and Bohke O 1987 Sol. Energy Mater. 1655

Davazoglou D and Donnadieu A 1987 Thin solid films 147131

Deb S K and Chopoorian J A 1966 J. Appl. Phys. 374818

Donnadieu A and Davazoglou D 1986 Proc. Third SPIE Symp., Innsbruck 65336

Eda K 1989 J. Solid State Chem. 83292

Lampert C M 1984 Sol. Energy Mat. 111

Yoshimura T, Watanabe M, Koike Y, Kiyoa K and Tanaka M 1983 Thin Solid Films 101141 